\title{
Flexible, Light-Weight Antenna at 2.4GHz for Athlete Clothing
}

\author{
Amir Galehdar and David V. Thiel* \\ Centre for Wireless Monitoring \& Applications \\ Griffith School of Engineering, Griffith University - Nathan \\ Queensland, 4111, Australia \\ E-mail: d.thiel@griffith.edu.au
}

\begin{abstract}
Linearly polarized rectangular patch antennas printed on light-weight cotton clothing are subject to both convex and concave bending. Changes in resonant frequency resulting from $\mathrm{H}$ plane bending are explained in terms of changes in effective length. Agreement between theory, practical and simulation results was observed. The resonant frequency changed by up to $8 \%$ for extreme bending although the bandwidth remains essentially unchanged at $4.5 \%$. A $16.8 \%$ bandwidth was achieved using a double U-Slotted patch which minimized the problem.
\end{abstract}

Key words: wearable antennas, patch antenna, dual slots, curved patch.

\section{Introduction}

Athlete monitoring during sporting events and training is of significant interest to coaches and television broadcasters. Coaches seek biomechanical and physiological information to make tactical changes during the event. Television broadcasters seek to inform viewers as the status of the athlete. Wearable antennas for these applications must include sufficient antenna gain to minimize battery size and sufficient range to cover the field of play (usually not less than $100 \mathrm{~m}$ line-of-sight).

In this paper the effects of concave and convex bending in $\mathrm{E}$ and $\mathrm{H}$ plane on wearable antennas are reported. An increase in the bend angle for a concave $\mathrm{H}$ plane increased the resonant frequency. This is thought to result from a decrease in effective length. Convex $\mathrm{H}$ plane bending has the opposite effect. These results were verified by simulation, experiment results and an approximate theory. Concave E plane bending was measured and explained by previous researchers $[1,2]$. An increase in bend angle decreased the resonant frequency.

This paper outlines the design of a $2.45 \mathrm{GHz}$ flexible patch antenna mounted on fabric having thickness $1.6 \mathrm{~mm}$ and relative permittivity $\mathcal{E}_{\mathrm{r}}=1.63$ (determined experimentally). The fabric adhesive used to fix the copper patch and ground plane increased the effective relative permittivity to 2.6. The basic design was built using flexible copper mesh as both the ground plane and the patch with fabric as the dielectric. Adhesive backed copper-coated non-woven nylon fabric is available from commercial suppliers for EMI suppression applications. 


\section{Resonant frequency}

The resonant frequency $f_{m n}$ of a planar, rectangular patch antenna can be determined by the width $W$ and length $L$ of the patch and the thickness $t$ and permittivity $\varepsilon_{\text {eff }}$ of the dielectric [3].

$$
f_{m n}=\frac{c}{2 \varepsilon_{e f f}^{1 / 2}}\left[\left(\frac{m}{L}\right)^{2}+\left(\frac{n}{W}\right)^{2}\right]^{1 / 2}
$$

where $m$ and $n$ are the mode numbers. When the patch antenna is curved in an arc along its length (H plane), $L=R \theta$ where $R$ is the radius of curvature and $\theta$ is the angle subtended by the patch length [2]. The angle is positive when the centre of curvature is closer to the ground plane and negative when it is closer to the patch. Fig 1 shows a positive $\theta$ bend.

E plane: Experimentally a decrease in resonant frequency was observed for increasing $\theta$. In the extreme case a $8 \%$ decrease in the resonant frequency in both positive and negative directions was measured (see fig.2 and 3).

$H$ plane: Experimentally the resonant frequency increases for positive $\theta$, and decreases for negative $\theta$ (see Fig. 3 and 4). These results are confirmed by HFSS simulation (see Fig.3). This is different from previous observations where no change was observed [5]. The change in resonant frequency was found to be $8 \%$ for the maximum bend index by measurements. A cylindrical bend is thought to be a more accurate approximation compared to V-shape bends in clothing [2]. Equation (1) can be modified to include variations in the effective length by assuming the new length lies along an arc midway through the dielectric material. Ignoring changes to the fringing fields, the new length can be written as:

$$
L_{e f f}=\left(\frac{L}{\theta}-\frac{t}{2}\right) \theta=L-\frac{t \theta}{2}
$$

and $L_{\text {eff }}$ becomes $L$ in (1) and $\theta$ is zero. For the dominant mode, this equation predicts variations in $f_{10}$ for bends along the length. Fig. 4 shows variations for a square patch $(L=45 \mathrm{~mm}, W=54 \mathrm{~mm})$ on $t=1.6 \mathrm{~mm}$ cotton fabric with relative permittivity $\varepsilon_{r}=$ 2.41 (influenced by the presence of the fabric adhesive). The fit is not strong, however the change reflects both increased and decreased frequency depending on whether the patch is concave or convex. It is anticipated that the non-linearity is due predominantly to a change in the mutual coupling between the radiating slots of the patch antenna. A practical solution to this antenna problem lies in the application of techniques to increase the bandwidth of the patch.

\section{Dual U slots}

An effective, well known, method of increasing the bandwidth of a planar, rectangular patch antenna is through the use of one or more $U$ shaped slots symmetrically located in the patch [3]. Fig 5. shows an optimized dual slot configuration with a probe feed 
located $5 \mathrm{~mm}$ from the centre on the plane of symmetry. The bandwidth increased to $16.8 \%$ and the antenna was found to function satisfactorily though all bend likely conditions.

\section{Conclusions}

A rectangular patch antenna using thin cotton fabric as a dielectric was subjected to bending along two orthogonal axes. Bending was found to substantially change the fundamental resonant frequency to such an extent that it no longer functioned within the desired band. The inclusion of two $U$ shaped slots in the patch increased the bandwidth substantially resulting in a flexible antenna which functions satisfactorily under most bend conditions. Some of this work was previously presented [4].

\section{References:}

[1] Tanaka, M. and Jang, J. H., "Wearable Microstrip Antenna", 2003 IEEE Antennas and Propagation Society International Symposium, Columbus, Ohio, USA, vol, 2, pp. 704-707, 2003.

[2] C.M. Krowne, "Cylindrical-rectangular microstrip antenna", IEEE Antennas \& Prop., vol 31, (1), pp. 194-198, Jan. 1983.

[3] Kumar, and K.P. Ray, Broadband microstrip antennas, Artech House: Boston, MA., 2003.

[4] A. Galehdar, and D.V. Thiel, "Patch antennas on light weight clothing experimental and theoretical investigations." $9^{\text {th }}$ Australian Symposium on Antennas, Sydney, p. 38, Feb. 2005.

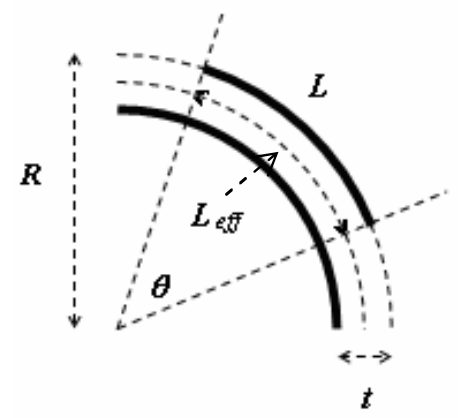

Fig 1: Positive $\theta$ bend along the length $L$ of the antenna (H plane). The curved dashed line indicates the new effective length of the patch $L_{\text {eff }}$ measured between the two radial lines.

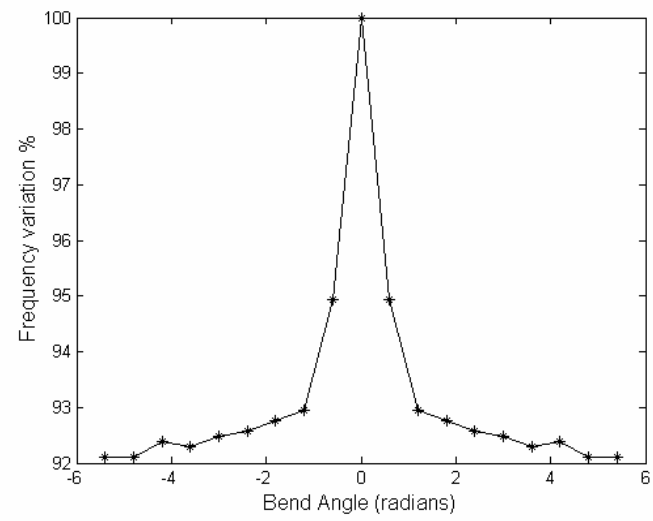

Fig 2: Experimental variation in $f_{10}$ for cylindrical bends along the patch width (E plane). 


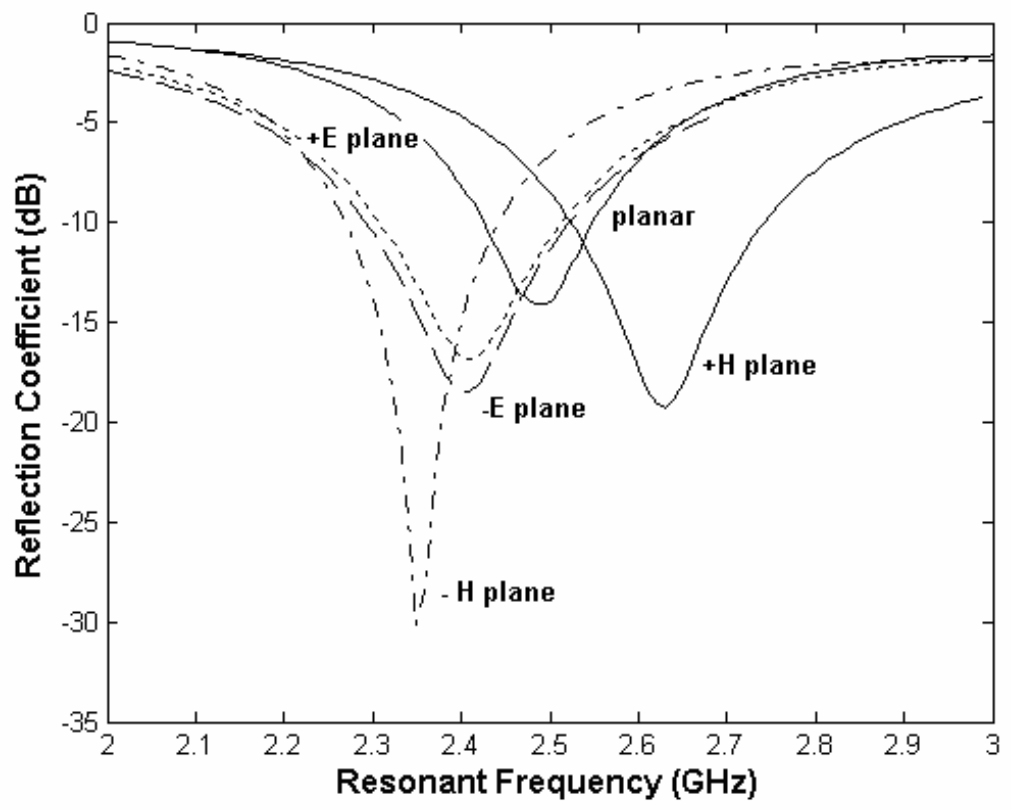

Fig.3 HFSS Simulation results for a planar patch $(\theta=0)$, and positive and negative curved patches in the $\mathrm{H}$ and $\mathrm{E}$ planes $(\theta= \pm 0.5$ radians $)$.

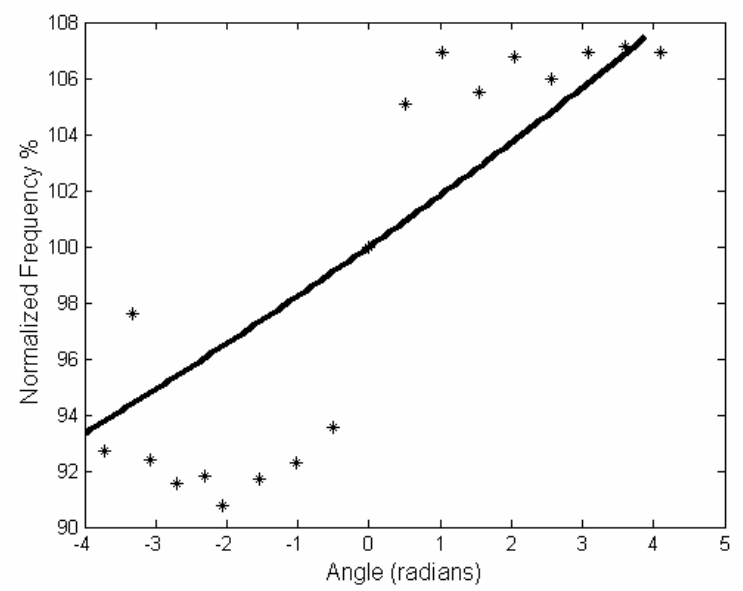

Fig 4: Experimental variation in $f_{10}$ for bends along the length. The line shows a theoretical prediction based on equations (1) and (2)

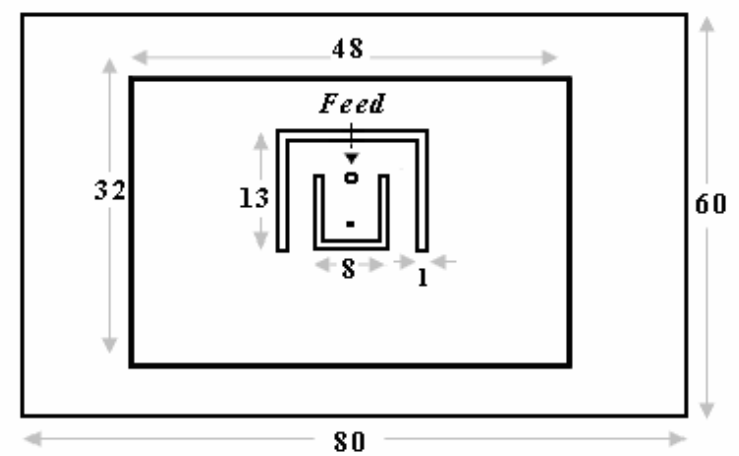

Fig 5: Double U-slotted patch showing dimensions in mms (fabric thickness is $1 \mathrm{~mm}$ ). 